Benchmarks

\section{Electrical stimulation of primary neonatal rat ventricular cardiomyocytes using pacemakers}

\author{
Ruben S.R.M. Martherus ${ }^{1,2}$, Volkert A. Zeijlemaker ${ }^{3}$, and \\ Torik A.Y. Ayoubi ${ }^{1,2, *}$ \\ ${ }^{1}$ Department of Clinical Genomics, Maastricht University, Maastricht, \\ The Netherlands, ${ }^{2}$ Cardiovascular Research Institute Maastricht, \\ Maastricht, The Netherlands, and 'Medtronic Bakken Research Center B.V., \\ Maastricht, The Netherlands
}

BioTechniques 48:65-67 (January 2010) doi 10.2144/000113308

Keywords: cell culture; cardiomyocyte; electrical stimulation; heart

*T.A.Y.A.'s current address is VIB Flanders Institute for Biotechnology MicroArray Facility, K.U. Leuven, Herestraat 49, P.O. Box 816, 3000 Leuven, Belgium.

The study of gene regulation in cardiac myocytes requires a reliable in vitro model. However, monolayer cultures used for this purpose are typically not exposed to electrical stimulation, though this has been shown to strongly affect cardiomyocyte gene expression. Based on pacemakers for clinical use, we developed an easy-to-use portable system that allows the user to perform electro-stimulation of cardiomyocyte cultures in standard tissue incubators without the need for bulky equipment. In addition, we present a refined protocol for culturing high-purity cardiomyocyte cultures with excellent contractile properties for a wide variety of applications.

Monolayer cultures of primary neonatal rat ventricular cardiomyocytes (NRVCMs) are a frequently employed model to study transcription regulation of cardiac-specific genes like atrial natriuretic factor (anf) nppa) and $\alpha$ myosin heavy chain (myb6) $(1-3)$. However, many of these studies do not incorporate electrical stimulation of cardiomyocytes, though it has been found that prolonged electrical stimulation improves cardiomyocyte morphology and function and that direct electrical pulsing increases transcript levels of cardiacspecific genes $(4,5)$.

Currently, in vitro stimulation of cardiomyocytes or engineered cardiac tissue is done using cardiac stimulators like those of Nihon Kohden (Tokyo, Japan) or Ion Optix (Milton, MA, USA), with parameters varying between 2 and $4 \mathrm{~ms}, 5$ and $11 \mathrm{~V} / \mathrm{cm}$, and 1 and $3 \mathrm{~Hz}(6-8)$. Such apparatuses have disadvantages: they need to be plugged in and their large dimensions make transport to other work areas (e.g., for microscope analysis) cumbersome. Furthermore, the machines normally have to be placed outside of incubators, since they are often not designed for use with high humidity and temperatures. As a result, structural modifications to the incubators may be required for connecting cables to run through door openings, which potentially compromises sterility and culture conditions.

We hypothesized that employing cardiac pacemakers normally used for heart patients would solve many of the aforementioned problems. These battery-powered pacemakers are small and are completely closed, so that the whole unit can be ethanolsterilized and placed inside incubators together with the cultures without the need for any modifications. Furthermore, the whole system together with cultures can easily be transported without interruption to the pulsing.

We cultured NRVCMs in commercially available chamber slides that can be used for a wide variety of purposes: live imaging, immunohistochemistry, reporter studies, and a single chamber provides sufficient material for gene expression analysis. Reusable polycarbonate lids were designed to fit the chamber slides and equipped with platinum electrodes running along opposite sides of each chamber. Small air channels in the lids enabled gas exchange (Figure 1). The electrode lids were connected to human pacemakers, models Kappa 931 and 401 (Medtronic, Minneapolis, MN, USA). The pacemakers were programmed using a Medtronic Vitatron 9790 programmer to give $7.5 \mathrm{~V}$ pulses (corresponding to $4.5 \mathrm{~V} / \mathrm{cm}$ ) with a duration of $1.5 \mathrm{~ms}$ at $1 \mathrm{~Hz}$, placing it in the same range as previous publications and resembling the levels required for exciting normal ventricular tissue (7-9). New pacemakers and programmers can be costly and may not be a feasible option for all researchers. However, a significant number of devices are explanted for various reasons. These explanted pacemakers can be obtained more cheaply and programmed with the help of cardiologists and may still be able to stimulate for several years.

The protocol employed for cardiomyocyte isolation and culture is a modified version of that described by Radisic et al. $(9,10)$. On day 1 , hearts were obtained from 0-3 day old Lewis rats locally bred at the animal facility of Maastricht University. The use of animals for this study was evaluated and approved by the institute's animal ethical committee. On day 2 , cardiomyocytes were pre-plated for $2 \mathrm{~h}$ an subsequently plated at $>30,000$ cells $/ \mathrm{cm}^{2}$ on plastic (Permanox, Lab-Tek; Nunc, Langenselbold, Germany) chamber slides and incubated overnight in high-serum medium supplemented with $10 \mu \mathrm{mol} / \mathrm{L}$ cytosine arabinoside (Ara-C; Cat. no. C1768; Sigma-Aldrich, St. Louis, MO, USA) to further decrease the number of actively dividing (mostly non-myocyte) cells $(11,12)$.

The Permanox chamber slides did not require coating since cardiomyocytes readily adhere to them. The culturing parameters were empirically determined through pilot experiments which revealed that the use of glass-based chamber slides with surface coatings like Histogrip (Invitrogen, Carlsbad, CA, USA) and especially Matrigel (BD Biosciences, San Jose, CA, USA) led to dramatic changes in cardiomyocyte cellular structure and function; cardiomyocytes remained rounded, resulting in diminished intercellular connections and fewer contractions (data not shown). At densities $<30,000$ cells $/ \mathrm{cm}^{2}$ without electrical 

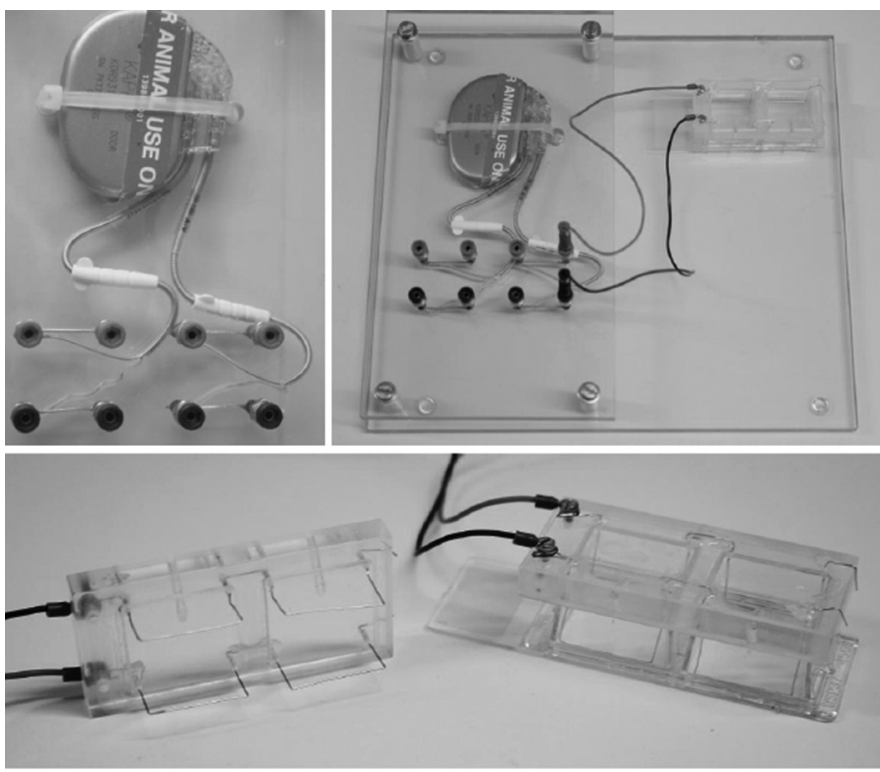

Figure 1. System for culturing electro-stimulated cardiomyocytes. Top left panel: Human pacemaker Medronic model KAPPA 931 fixed to a polycarbonate platform. Ventricular and atrial leads are connected to two separate pairs of sockets enabling electro-stimulation of two series with different pulse characteristics. Top right panel: Overview of pulsing system stimulation. Bottom panel: Reusable polycarbonate electrode lids designed to fit commercial dual-chamber slides.
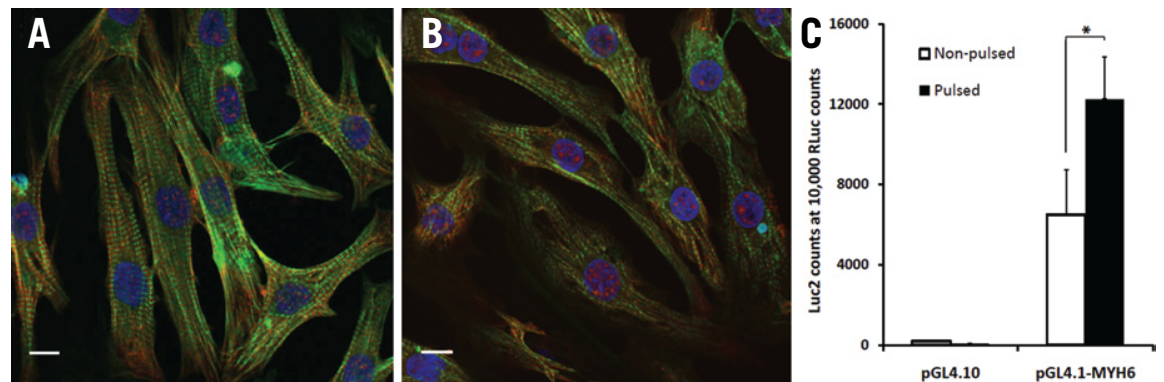

Figure 2. Gene expression in cultures of electro-stimulated cardiomyocytes. Confocal microscopy images of cardiomyocytes after 3 days of electric stimulation (A) and without stimulation (B). Cells were cultured and fixed on chamber-slides and stained with mouse antibody for rat cardiac specific troponin-I and FITC-labeled goat-anti-mouse. Actin was stained with Alexa-494 labeled phalloidin and nuclei with DAPI. Scale bar indicates $10 \mu \mathrm{m}$. (C) Transcriptional activity of a 2-kb human MYH6 promoter fragment in cultures with and without stimulation 3 days post-transfection. Cells were transfected with equal molar amounts of reporter plasmids and $10 \mathrm{ng}$ of pGL4.75 CMV- luciferase reporter vector. ${ }^{*}$ indicates $\mathrm{p}$-value $=0.03$.

\section{PRECISION BIOTECH Optics.}

- UV Lenses - Wide Selection of Coatings

- UV Filters - High Transmission OD 6 Rejection

- Request your FREE catalog!

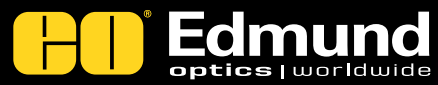

800.363.1992 | www.edmundoptics.com stimulation, the cells displayed no contractions and remained round. Over $50 \%$ of these cells died within 2 days of culture, most likely because they were not able to form the connections required for intercellular signaling.

The resulting cultures consisted of $>95 \%$ cardiomyocytes (purity of cultures can be observed in Figure 2). On day 3, the medium was replaced with Ara-C-free high-serum medium since Ara-C was shown to directly interfere with the contractile activity of the cells (data not shown). For transfection studies, cells can be transfected at this time. We obtained reproducible results for luciferase reporter constructs with the transfection reagent Fugene HD (Roche) using the manufacturer's protocols when transfecting cells in high-serum medium. In our studies we employed transfection studies to examine the promoter sequences of cardiac genes. A Myb6 promoter-luciferase reporter construct was transfected into cardiomyocytes which subsequently were electrostimulated (Figure 2). The behavior of the luciferase reporter construct is similar to that of the endogenous $M y b 6$; the activation observed in Figure 2 is believed to be electrical pulse-dependent.

Typical spontaneous contractions normally observed in cardiomyocytes became visible on day 4 , in the absence of electrical stimulation. From day 4 onwards, culture medium was refreshed every $24 \mathrm{~h}$ with differentiation medium (DMEM, 2\% horse serum, 1\% HEPES, gentamycin $0.10 \mathrm{mmol} / \mathrm{L}$, Fungizone $0.22 \mathrm{nmol} / \mathrm{L}$ ) to avoid starvation of the cells. Serum concentration was shown to directly affect formation of sarcomeric structures and contractile activity. This was to be expected since serum levels directly modulate serum response factor, a key cardiac transcription factor $(13,14)$. Empirically, we found normal differentiation and frequent spontaneous contractions in stimulated and non-stimulated cultures to be maintained at serum levels of $2 \%$ even after several days of culture. The best results for electro-stimulation were obtained when starting stimulation on day 4 together with the first addition of differentiation medium, thereby allowing for a recovery time of several days similar to that previously described (6).

We present herein a refined protocol for culturing NRVCMs, and a pacemakerbased pulsing system whose inherent characteristics renders it possible to place the whole system inside any standard incubator. This makes it much more convenient to use than conventional pulsing equipment. The electro-stimu- 
lated NRVCMs can be used for many applications including reporter assays - an interesting combination that, to our knowledge, has so far remained uninvestigated.

\section{Acknowledgments}

This work is supported by a grant from the School of Life Sciences, Transnationale Universiteit Limburg.

\section{Competing interests}

The authors declare no competing interests.

\section{References}

1. Kim, T.G., J. Chen, J. Sadoshima, and Y. Lee. 2004. Jumonji represses atrial natriuretic factor gene expression by inhibiting transcriptional activities of cardiac transcription factors. Mol. Cell. Biol. 24:10151-10160.

2. Kim, T.G., J. Jung, M.R. Mysliwiec, S. Kang, and Y. Lee. 2005. Jumonji represses alpha-cardiac myosin heavy chain expression via inhibiting MEF2 activity. Biochem. Biophys. Res. Commun. 329:544-553.

3. Ojamaa, K., A.M. Samarel, and I. Klein. 1995. Identification of a contractile-responsive element in the cardiac alpha-myosin heavy chain gene. J. Biol. Chem. 270:31276-31281.

4. Holt, E., P.K. Lunde, O.M. Sejersted, and G. Christensen. 1997. Electrical stimulation of adult rat cardiomyocytes in culture improves contractile properties and is associated with altered calcium handling. Basic Res. Cardiol. 92:289-298.

5. McDonough, P.M. and C.C. Glembotski. 1992. Induction of atrial natriuretic factor and myosin light chain-2 gene expression in cultured ventricular myocytes by electrical stimulation of contraction. J. Biol. Chem. 267:11665-11668.

6. Radisic, M., H. Park, H. Shing, T. Consi, F.J. Schoen, R. Langer, L.E. Freed, and G. Vunjak-Novakovic. 2004. Functional assembly of engineered myocardium by electrical stimulation of cardiac myocytes cultured on scaffolds. Proc. Natl. Acad. Sci. USA 101:18129-18134.

7. Rybkin, I.I., D.W. Markham, Z. Yan, R. Bassel-Duby, R.S. Williams, and E.N. Olson. 2003. Conditional expression of SV40 T-antigen in mouse cardiomyocytes facilitates an inducible switch from proliferation to differentiation. J. Biol. Chem. 278:15927-15934.

8. Yonemochi, H., S. Yasunaga, Y. Teshima, N. Takahashi, M. Nakagawa, M. Ito, and T. Saikawa. 2000. Rapid electrical stimulation of contraction reduces the density of beta-adrenergic receptors and responsiveness of cultured neonatal rat cardiomyocytes. Possible involvement of microtubule disassembly secondary to mechanical stress. Circulation 101:2625-2630.

9. Radisic, M., M. Euloth, L. Yang, R. Langer, L.E. Freed, and G. Vunjak-Novakovic. 2003. High-density seeding of myocyte cells for cardiac tissue engineering. Biotechnol. Bioeng. 82:403-414.

10. Radisic, M., A. Marsano, R. Maidhof, Y. Wang, and G. Vunjak-Novakovic. 2008. Cardiac tissue engineering using perfusion bioreactor systems. Nat. Protocols 3:719-738.

11. de Vries, J.E., M.M. Vork, T.H. Roemen, Y.F. de Jong, J.P. Cleutjens, G.J. van der Vusse, and M. van Bilsen. 1997. Saturated but not mono-unsaturated fatty acids induce apoptotic cell death in neonatal rat ventricular myocytes. J. Lipid Res. 38:1384-1394.

12.Smeets, P.J.H., B.E.J. Teunissen, A. Planavila, H. de Vogel-van den Bosch, P.H.M. Willemsen, G.J. van der Vusse, and M. van Bilsen. 2008. Inflammatory pathways are activated during cardiomyocyte hypertrophy and attenuated by peroxisome proliferator-activated receptors PPAR-alpha and PPAR-delta. J. Biol. Chem. 283:29109-29118.

13. Miano, J.M., X. Long, and K. Fujiwara. 2007. Serum response factor: master regulator of the actin cytoskeleton and contractile apparatus. Am. J. Physiol. Cell Physiol. 292:C70-C81.

14. Treisman, R. 1986. Identification of a protein-binding site that mediates transcriptional response of the c-fos gene to serum factors. Cell 46:567-574.

Received 1 September 2009; accepted 20 October 2009.

Address correspondence to Torik Ayoubi, VIB Flanders Institute for Biotechnology MicroArray Facillity, K.U. Leuven, Herestraat 49, P.O. Box 816,3000 Leuven, Belgium.email: torik.ayoubi@vib.be

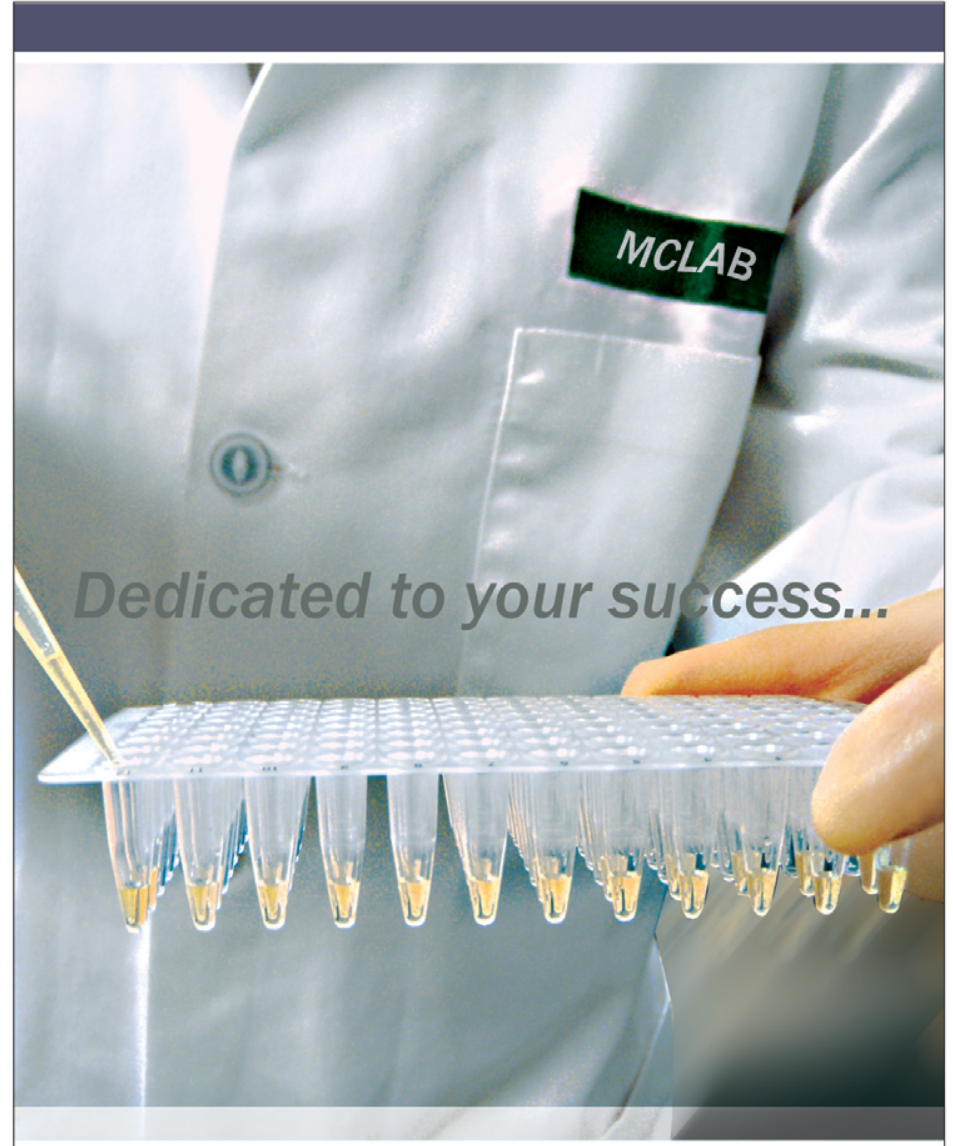

$M C L A B$

\section{your cloning lab.}

$\checkmark$ Check the savings - \$250/clone; cheaper than your

in-house operations.

$\checkmark$ No-nonsense - verification of clones through sequencing.

$\checkmark$ Reliable - with our revolutionary cloning technology, the Choo-Choo ${ }^{\circledR}$ cloning system, any construct is possible. Get high-throughput single or multiple DNA fragment cloning in 2-7 days.

$\checkmark$ Flexible - restriction enzyme independence and able to use any customer or commercial vectors.

$\checkmark$ Seamless - no extra amino acid at the insert-vector junction.

$\checkmark$ Extendable-subsequent in-house production of any amount of plasmid DNA possible. 